\title{
Active Control of Counter-Rotating Open Rotor Interior Noise in a Dornier 728 experimental aircraft
}

\author{
Thomas Haase, Oliver Unruh \\ Institute of Composite Structures and Adaptive Systems, German Aerospace Center \\ (DLR), Lilienthalplatz 7, Braunschweig, Germany \\ Institut für Adaptronik und Funktionsintegration, Technical University Braunschweig, \\ Langer Kamp 6, Braunschweig, Germany \\ Stephan Algermissen, Martin Pohl \\ Institute of Composite Structures and Adaptive Systems, German Aerospace Center \\ (DLR), Lilienthalplatz 7, Braunschweig, Germany
}

\begin{abstract}
The fuel consumption of future civil aircraft needs to be reduced because of the $\mathrm{CO}_{2}$ restrictions declared by the European Union. A consequent lightweight design and a new engine concept called counter-rotating open rotor (CROR) are seen as key technologies in the attempt to reach this ambitious goals. Bearing in mind that CROR engines emit very high sound pressures at low frequencies and that lightweight structures have a poor transmission loss in the lower frequency range, these key technologies raise new questions in regard to acoustic passenger comfort. One of the promising solutions for the reduction of sound pressure levels inside the aircraft cabin are active sound and vibration systems. So far, active concepts have rarely been investigated for a CROR pressure excitation on complex airframe structures. Hence, the preliminary study presented in this paper shows how an active control system can influence the sound radiation of a complex airframe structure under a CROR pressure excitation and also addresses the open questions on the way towards its realisation. In this phase, an active feedforward control system is investigated in a fully equipped Dornier 728 experimental prototype aircraft. In particular, the sound transmission through
\end{abstract}

Email address: thomas.haase@dlr.de (Thomas Haase) 
the airframe, the coupling of classical actuators into the structure and the performance of the active vibration control system with different error sensors are investigated. It can be shown that the active control system achieves a reduction up to $5 \mathrm{~dB}$ at several CROR frequencies but also that a better performance could be achieved through further optimisations.

Keywords: CROR, feedforward control, active vibration control, airframe structures

\section{Introduction}

The fuel consumption of future civil aircraft needs to be reduced because of the $\mathrm{CO}_{2}$ restrictions declared by the European Union. A consequent lightweight design and a new engine concept called counter-rotating open rotor (CROR) are seen as key technologies in the attempt to reach this ambitious goals. Because of the high sound pressure levels emitted by the CROR engines and the poor transmission loss of lightweight structures in the lower frequency range, the combination of these technologies is very challenging when it comes to an aircraft's interior noise [1], [2].

In order to improve the transmission loss at the CROR blade passing frequencies (BPF), active concepts offer a lightweight-compliant solution to interior noise problems. For example, a feedback control system is investigated by the authors in [3]. This is why, during the last 20 years, many active concepts such as active noise control (ANC), active vibration control (AVC) and active structural acoustic control (ASAC) have been investigated in order to solve sound radiation problems; for examples see [4] and [5].

ANC is a widely known approach for the active reduction of noise levels. A lot of theoretical and experimental studies for ANC in cavities (see for example [6]) and double wall cavities [7] have been conducted. Even a very complex load master area of the Airbus A400M has been controlled with an ANC system [8] and Cabell et. al. [9] conducted an experimental flight test of an ANC system..

Another approach is an ASAC system. While the acronym ASAC is frequently used, it also conveys different meanings. Therefore, the authors deem it important to note that their understanding of an ASAC system is as follows: An ASAC system works with a controller that uses structural sensors, structural actuators and an acoustical post processing to directly influence the radiated power as the controller cost function. A lot of work 
focuses on the reduction of the sound radiation of plates [10], [11] and also of more complex structures like a car wind shield [12] and a truck oil pan [13]. In contrast to the studies and the ASAC definition mentioned above, this paper presents an AVC system that reduces the flexural vibration at local structural error sensors with structural actuators in order to indirectly reduce the sound power as it is desirable to reduce the vibrations on and the sound radiation of the airframe [14]. Some previous work of Grewal et al. shows promising results for an active control system reducing multisinusoidal excitations [15]. The authors also published a preliminary study on a stiffened carbon fibre reinforced plastic (CFRP) panel mounted in a transmission loss facility which shows promising results, too [16]. Reviewing the literature reveals that less work has been done on the development of AVC systems for a real aircraft with an airframe, windows and joints that is excited by a synthesised CROR sound pressure field. Due to the structural complexity and the realistic excitation, new challenges for an active control system arise beyond the state of the art.

Therefore, this study will demonstrate the possibility of using an AVC system to improve the low frequency transmission loss of an airframe excited by a synthetic CROR sound pressure field. Hence, the objectives addressed in this paper are:

- Investigation of the sound transmission of the CROR sound pressure field through the airframe

- Experimental test of typical control actuators regarding their coupling capabilities

- Realisation of an AVC system in order to improve the transmission loss at the CROR frequencies

The paper is structured as follows: First, the Dornier 728 experimental prototype aircraft and the test section (part of the airframe equipped with the active system) are described. In the next section, the theoretical basics for the feedforward controller design are explained and, subsequently the experimental control results are presented and discussed. The paper ends with a conclusion and an outlook to future research. 


\begin{tabular}{|l|l|l|l|l|l|l|l|}
\hline Index & 1 & 2 & 3 & 4 & 5 & 6 & 7 \\
\hline Frequency $[\mathrm{Hz}]$ & 119.6 & 149.3 & 268.5 & 388.1 & 417.8 & 507.5 & 537.2 \\
\hline
\end{tabular}

Table 1: CROR blade passing frequencies

\section{Testbed Dornier 728}

The DLR's grounded experimental prototype aircraft Dornier 728 makes it possible to carry out research on a fully equipped airframe and cabin. It is a regional aircraft with 70 seats and an operational range of up to $4700 \mathrm{~km}$. The Dornier 728 has an aluminium fuselage with stiffeners which are classically connected by rivets. The test section is located at the end of the cabin and encompasses two windows (see Figure 1). In order to excite the airframe with a CROR pressure field, a 112-channel loud speaker array is used. The speaker array is placed closely in front of the fuselage (approximately 150 $\mathrm{mm}$ ), which guarantees that the array is operating in the nearfield. This is also facilitated by the fact that the curvature of the speaker array can be adjusted to the curvature of the Dornier 728. Figure 2 exemplarily shows the speaker array in front of the Dornier 728 fuselage; it should be noted, however, that for measurements the speaker array is much closer to the aircraft fuselage.

For reasons of brevity, the description of the sound field synthesis is not given here. Detailed information about the sound field synthesis can be found in [16] and [17]. The addressed CROR BPFs are presented in Table 1.

In this study, the performance of an active feedforward control system on a real aicraft structure will be investigated. Furthermore, limitations and optimisation potentials will be identified. In this preliminary stage aspects of robustness or different flight scenarios are not concerned.

In summary, the instrumentation of the test section consists of 25 accelerometers used as error sensors and 12 inertial actuators. The global vibration of the test section is measured with a laser scanning vibrometer (LSV) and the radiated sound power with a sound intensity probe. In order to reduce antialiasing and to reconstruct the zero-order hold outputs of the digital signal processor (DSP), the error sensors and the actuators are passed through a low-pass filter. A detailed list of the experimental hardware is given in Table 2. Further information about the actuator and sensor placement is given in the following paragraphs.

Firstly, it has to be decided which type of actuator needs to be used to con- 


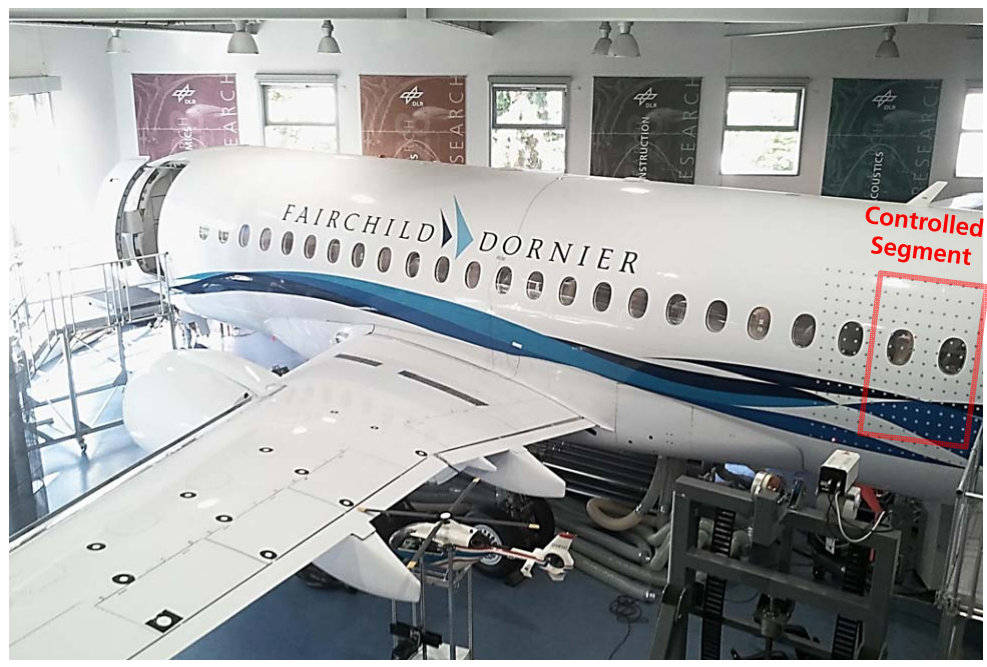

(a) Outside

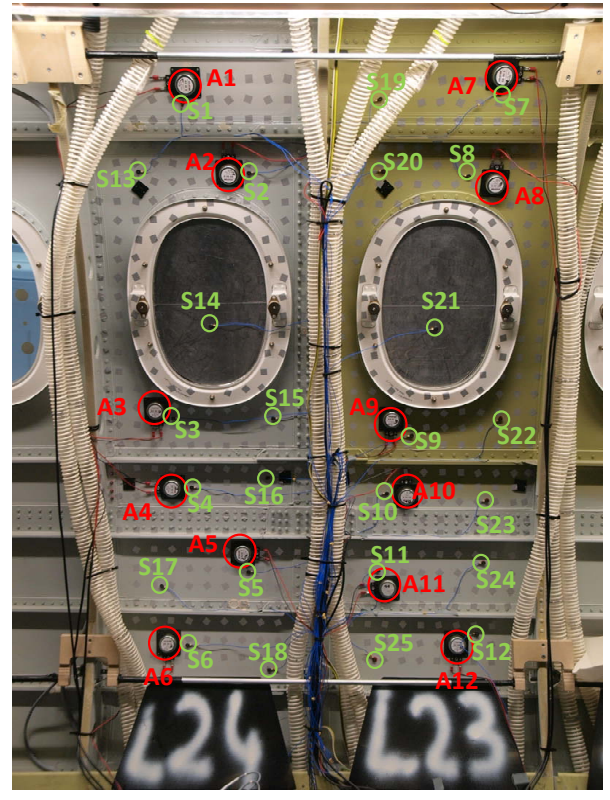

(b) Cabin

Figure 1: Dornier 728 experimental prototype aircraft (a) and view on the AVC control system with all sensors and actuators (b) 


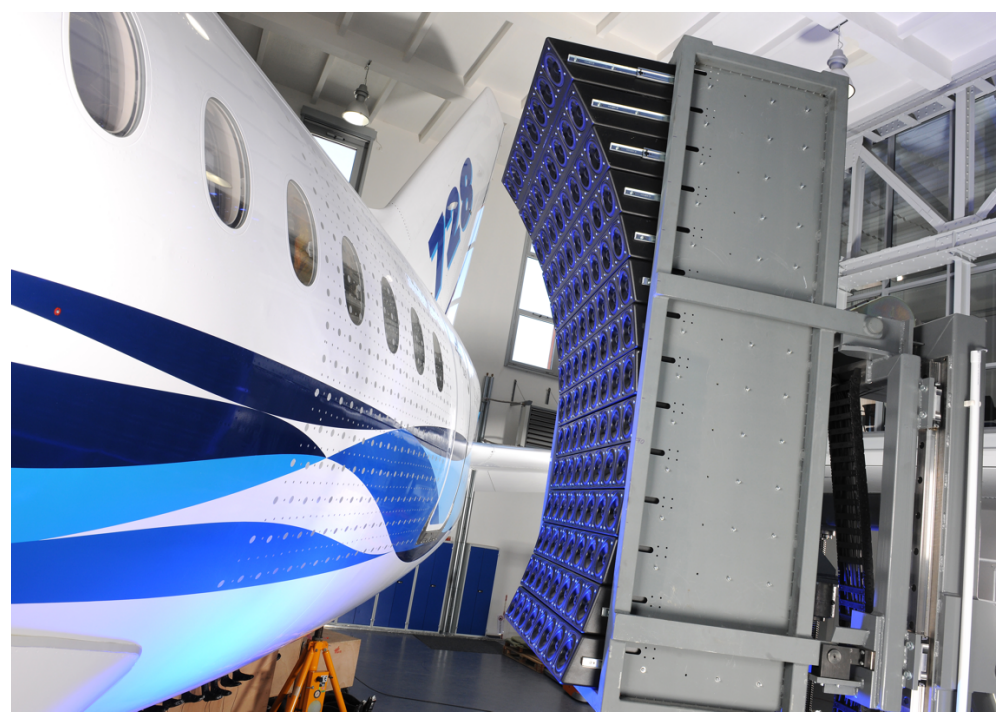

Figure 2: View on fuselage with speaker array

\begin{tabular}{l|l|l} 
Hardware & Type & Add. Information \\
\hline Real-time system & dSpace DS1006 & $F_{s}=2000 \mathrm{~Hz}$ \\
Laser scanning vibrometer & Polytec PSV 400 & - \\
Accelerometer & PCB 352A24 & $m=0.8 \mathrm{~g}$ \\
Exciter & Visaton EX60 & $m=120 \mathrm{~g}$ \\
Piezopatch actuator & DuraActP-876.A15 & $m=10 \mathrm{~g}$ \\
Low-pass filter & Kemo Card Master 255G & $F_{c}=480 \mathrm{~Hz}$ \\
Sound intensity probe & Bruel \& Kjaer & Type 3599
\end{tabular}

Table 2: Experimental hardware 
trol the flexural vibration induced by the CROR excitation. Therefore, the most commonly used inertial actuators and piezoceramic patch actuators, as shown in Figure 4b, are compared and evaluated in terms of the coupling performance in the fuselage structure. For a better understanding of the experiments, two block diagrams presented in Figure 3 show the measurement arrangement. In order to receive comparable results, the amplifiers of the piezopatch and the inertial actuators are included in the frequency response functions (FRFs) in such a way that the input is the excitation signal (bandlimited pseudo random noise) of the laser scanning vibrometer (LSV) and the output is the measured velocity. The gain of the amplifiers is chosen in such a way that both actuators works in their allowable range if the LSV generator excitation voltage is limited to $\pm 10 \mathrm{~V}$. The spatially averaged magnitudes of the FRFs of the panel section are compared in Figure 4. It can easily be seen that the inertial actuators perform much better in the lower frequency range than the flat piezopath actuators. Due to the fact that only the first seven CROR BPFs between 100 and $540 \mathrm{~Hz}$ are considered in this study, the inertial actuators are used throughout this study.

For a reasonable actuator placement, the vibration patterns of the first seven CROR frequencies are superposed. For that reason the velocities at the CROR frequencies are measured at the LSV meas grid (650 points equally distributed over the control surface). The inertial actuators are now placed in the maxima of the amplitudes and distributed over the entire surface in order to improve the controllability, see Figure 5. No less than 12 inertial shakers are placed in an area of $1.5 \mathrm{~m}^{2}$. High vibration amplitudes can also be observed in the window area, but it is a technically non-realisable scenario to mount actuators on the window and the window frame.

The accelerometers which can be later used as error sensors for the AVC system are empirically placed over the control surface. Finally, 12 sensors are collocated with the actuators and the rest are distributed over the control surface in order to achieve a good observability.

\section{Causal Feedforward Control}

A CROR engine typically emits harmonic tones which correlate with the rotational frequencies of the rotors. Apart from the basic harmonics there are several interaction tones in the CROR spectra. Nevertheless, a reference signal is easily accessible from the tachometer, which is why a CROR excitation is a typical application scenario for an active adaptive feedforward control 


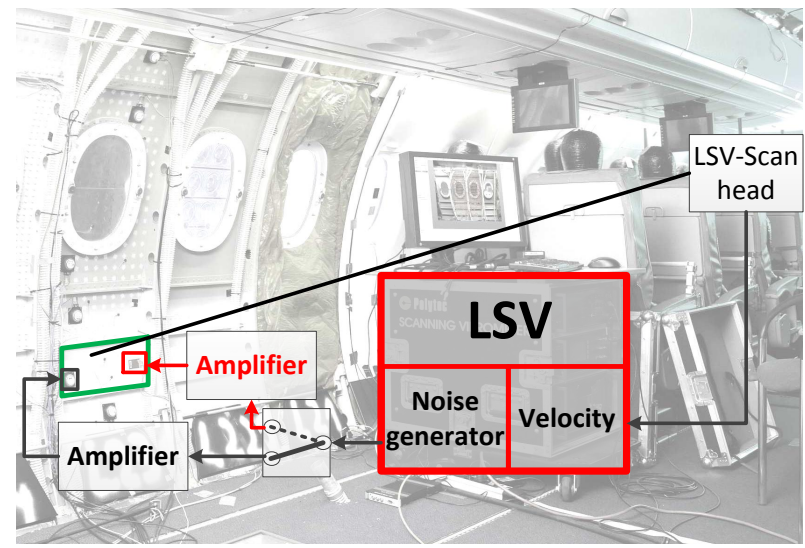

(a) Actuator coupling

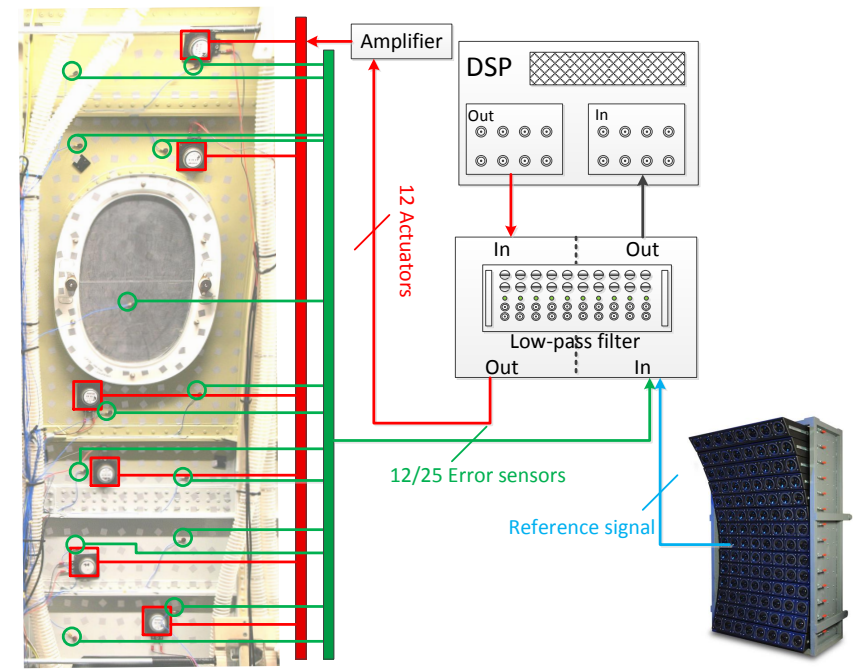

(b) Control system

Figure 3: Flow charts of the experimental measurements 


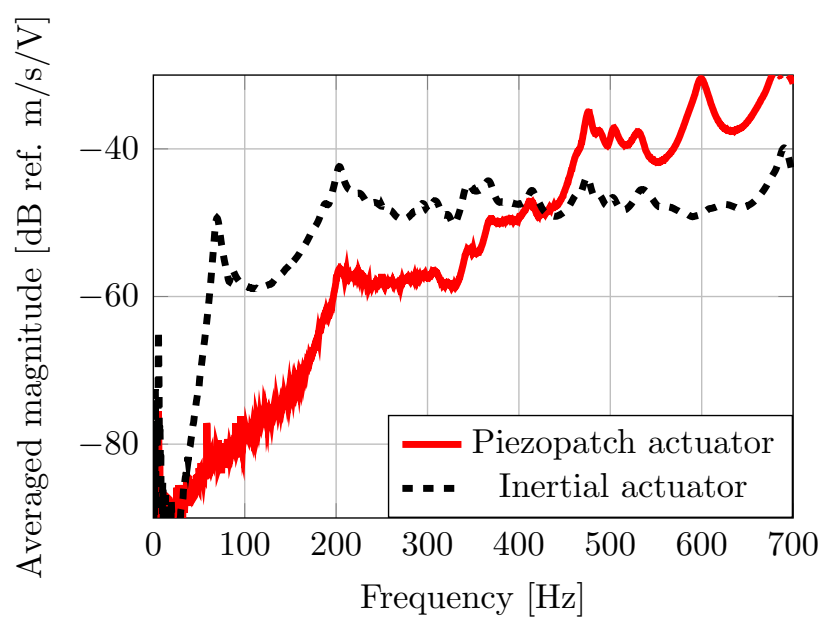

(a) Magnitude

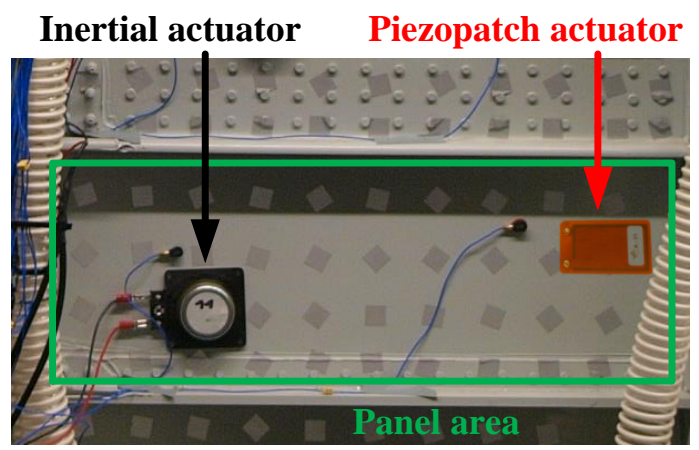

(b) Test set-up

Figure 4: Magnitudes and arrangement of the different actuators 


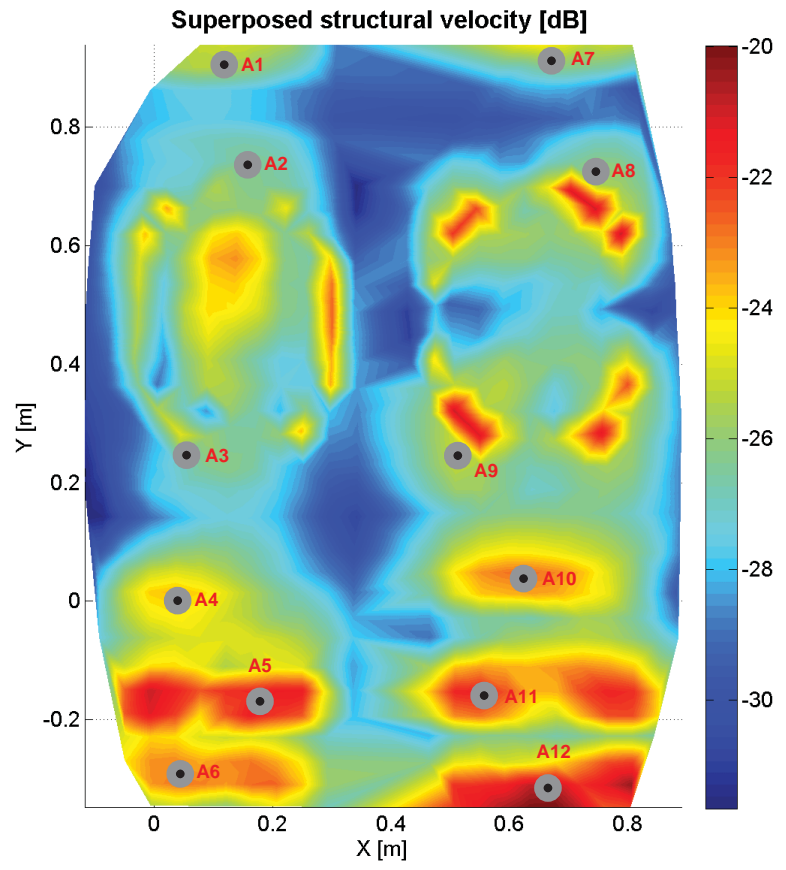

Figure 5: Superposed vibration patterns of the test section for the first seven CROR frequencies and actuator positions 

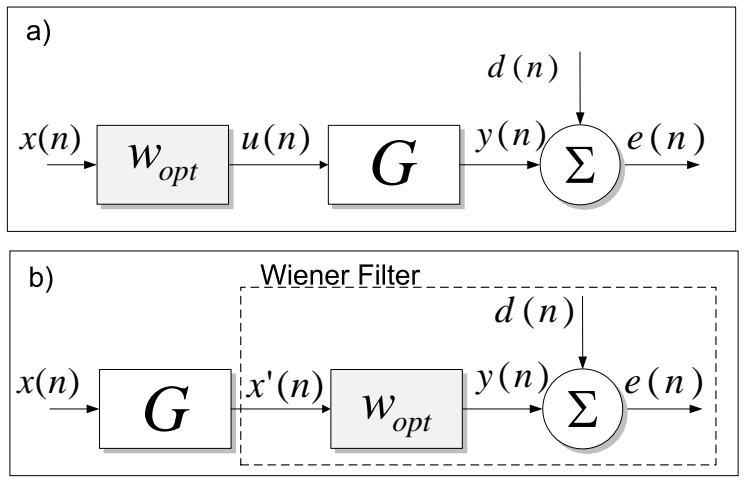

Figure 6: Block diagram of a feedforward control system for physical implementation (a) and for filter calculation (b)

system. The advantage of a feedforward controller lies in its stability (90 degree phase margin) and its robustness (adaptive algorithm). Furthermore, the reference signal is not disturbed by any feedback effect of the active feedforward controller, which influences the stability of the active control system [18]. For the evaluation of the performance of an adaptive feedforward controller in steady state without the need to wait until convergence an optimal feedforward controller is implemented in this study. This optimal feedforward controller exactly predicts the performance of an adaptive feedforward controller for stationary excitations [19]. For a better understanding, a brief description of a single-input single-output (SISO) optimal causal feedforward controller is given here; for detailed information see [19].

A typical block diagram of a feedforward controller is presented in Figure 6. Assuming that the optimal filter $\mathbf{w}_{\mathbf{o p t}}$ and the secondary path transfer function $\mathbf{G}$ are linear and time invariant, the order of the systems can be reversed [19]. Reversing $\mathbf{G}$ and $\mathbf{w}_{\mathbf{o p t}}$ allows the application of the classical Wiener filter theory by using the filtered reference signal $x^{\prime}(n)$. This filtered reference signal is determined for the SISO case by

$$
x^{\prime}(n)=\sum_{r=0}^{R-1} g_{r} x(n-r),
$$

where $x(n)$ is the reference signal at the discrete time $n, g_{r}$ are the coefficients of a finite impulse response (FIR) filter representing the secondary path and 
$R$ is the length of the secondary path FIR filter. Referring to Figure $6 \mathrm{~b}$ and assuming a quadratic cost function $J$ of the error signals

$$
J=E\left[e(n)^{T} e(n)\right]
$$

where $E[\cdot]$ is expectation operator, the Wiener filter can be calculated by using the autocorrelation matrix $\mathbf{R}_{\mathbf{x}^{\prime} \mathbf{x}^{\prime}}$ of the filtered reference signal and the crosscorrelation vector $\mathbf{r}_{\mathbf{x}^{\prime} \mathbf{d}}$ of the filtered reference signal and the disturbance signal $d(n)$.

$$
\mathbf{w}_{\mathbf{o p t}}=\mathbf{R}_{\mathbf{x}^{\prime} \mathbf{x}^{\prime}}{ }^{-1} \mathbf{r}_{\mathbf{x}^{\prime} \mathbf{d}}
$$

Now writing the samples of the filtered reference signal as a vector of the length $L$

$$
\mathbf{x}^{\prime}(n)=\left[x^{\prime}(n) x^{\prime}(n-1) \cdots x^{\prime}(n-L+1)\right],
$$

the auto- and crosscorrelation matrix of the filtered reference signal can be described by

$\mathbf{R}_{\mathbf{x}^{\prime} \mathbf{x}^{\prime}}=E\left[\mathbf{x}^{\prime \mathbf{T}}(n) \mathbf{x}^{\prime}(n)\right]=\left(\begin{array}{cccc}r_{x^{\prime} x^{\prime}}(0) & r_{x^{\prime} x^{\prime}}(1) & \ldots & r_{x^{\prime} x^{\prime}}(L-1) \\ r_{x^{\prime} x^{\prime}}(1) & r_{x^{\prime} x^{\prime}}(0) & \ldots & r_{x^{\prime} x^{\prime}}(L-2) \\ \vdots & \ldots & \ddots & \vdots \\ r_{x^{\prime} x^{\prime}}(L-1) & r_{x^{\prime} x^{\prime}}(L-2) & \ldots & r_{x^{\prime} x^{\prime}}(0)\end{array}\right)$

with

$$
r_{x^{\prime} x^{\prime}}(k)=E\left[x^{\prime}(n) x^{\prime}(n-k)\right],
$$

where $E[\cdot]$ is the expectation operator and the crosscorrelation vector is

$$
\mathbf{r}_{\mathbf{x}^{\prime} \mathbf{d}}=E\left[\mathbf{x}^{\prime \mathbf{T}}(n) d(n)\right]=\left[\begin{array}{llll}
r_{x^{\prime} d}(0) & r_{x^{\prime} d}(1) & \ldots & r_{x^{\prime} d}(L-1)
\end{array}\right]^{T} .
$$

The performance of the system can be evaluated in terms of the residual error signal

$$
e(n)=d(n)-\sum_{r=0}^{R-1} g_{r} \mathbf{w}_{\mathbf{o p t}}^{\mathbf{T}} \mathbf{x}(n-r),
$$

where $\mathbf{x}(n)$ is the reference signal vector of length L described by

$$
\mathbf{x}(n)=\left[\begin{array}{llll}
x(n) & x(n-1) & \ldots & x(n-L+1)
\end{array}\right]^{T} .
$$

It can easily be seen from Equation 3 that the optimal controller weights can be calculated off-line and implemented into the controller as long as the following steps are taken: 
- Identification of the secondary path

- Synchronous measurement of the reference signal and the disturbance signal

Once the controller weights have been calculated, the error signal reduction can be evaluated by measuring the performance with the controller switched on and off.

\section{Experimental results}

In this section, the experimental results are presented. First of all, an acoustic characterisation of the test section is conducted. Afterwards, the coupling of each inertial actuator into the whole test section is analysed (the comparison with the piezopatch actuator was only for a panel section), followed by the identification of the secondary path. Finally, the feedforward control results and a discussion are presented.

\subsection{Acoustic characterisation of the test section}

In order to investigate the sound transmission through the Dornier 728 airframe an intensity contour plot is measured. In order to achieve this the intensities of the first seven CROR frequencies are superposed and shown in Figure 7. It can be seen that the noise is transmitted efficiently through the lower part of test section. This fact was also clearly audible during the measurements and is related to the vibration maxima in the lower part of the test section which can be seen in Figure 5.

Surprisingly, high sound intensity values are not observed in the window area, as was to be expected from the vibration amplitude distributions shown in Figure 5. A reason for this could be the frame, which adds additional mass and stiffness in this area and therefore increases transmission loss. Yet, windows typically have a poor transmission loss in the lower frequency range [20], and for this reason it is a surprising result.

\subsection{Actuator performance}

For the investigation of the actuator performance, FRFs from the actuators to the sensor grid of the LSV are measured sequentially one after another. For each actuator, a LSV measurement is done and the averaged magnitude (from $1 \mathrm{~Hz}$ to $1 \mathrm{kHz}$ ) is plotted over the whole test area. Figure 8 exemplarily shows the performance of two actuators. It can easily be seen 


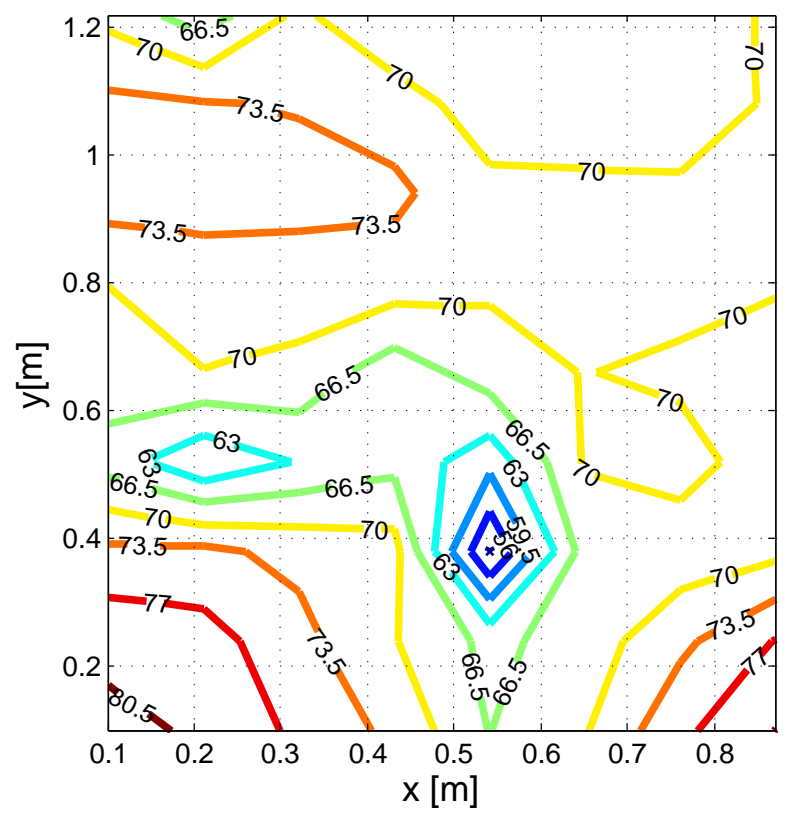

Figure 7: Superposed intensities of the test section for the first seven CROR BPFs (Unit of the scatter plot $[\mathrm{dB}$ ref $1 \mathrm{pW}]$ )

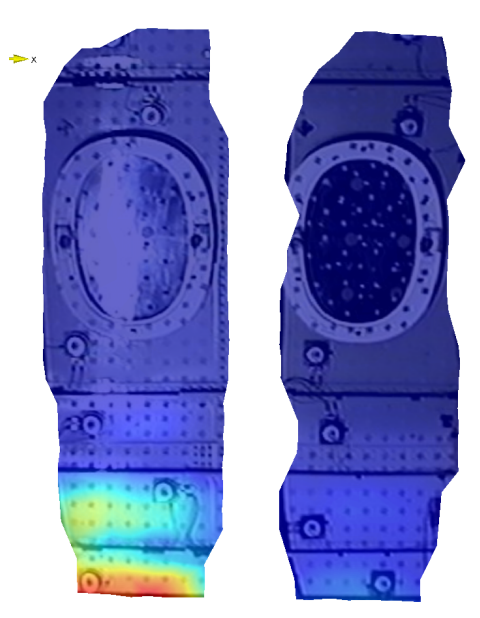

(a) Actuator A6
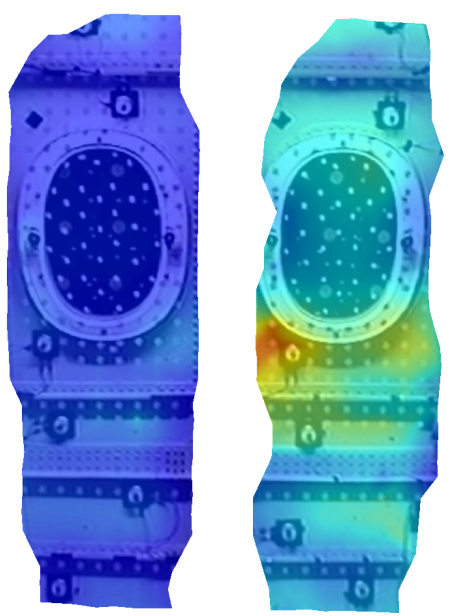

(b) Actuator A9

Figure 8: Performance of the actuators A6 and A9 over the whole test section 
that the coupling of the actuators in the fuselage structure is limited to the area of their location. Especially the actuators below the window section act only in the fuselage skin between the stiffeners. As an exception Actuator A9 in the window section acts more globally. In summary, the performance of the actuators is mainly limited to the region between two vertical stiffeners. This is caused by the high structural damping induced by the rivets and stiffeners. For active control purposes, this can be an advantage if a decentralised system for a larger control area is considered because the interaction of the control systems between two vertical stiffeners is small.

\subsection{System identification}

Referring to Equation 1 a precise secondary path model $\mathbf{G}$ is needed to calculate the filtered reference signals. Therefore, a multi-reference test is conducted to derive a state-space model of the secondary path. The actuators are driven by band-limited uncorrelated white noise signals and the sensor signals are sampled simultaneously. In order to enhance the signal-to-noise ratio of the identification process, the oversampling method is used. After the measurement of the time data, the input and output signals are postprocessed with a subspace-based identification algorithm [21]. The bandwidth of the system model is limited to $1000 \mathrm{~Hz}$. The singular values of the identified state-space and the measured frequency response functions for this range can be seen in Figure 9. It can easily be seen that the signal to noise ratio is not sufficient to identify a precise model in the frequency range from $0 \mathrm{~Hz}$ to $70 \mathrm{~Hz}$ due to the reduced sensitivity of the actuators and sensors in the lower frequency range. However, there is a high congruity between in the range of the addressed CROR BPFs from $110 \mathrm{~Hz}$ to $540 \mathrm{~Hz}$. Above $540 \mathrm{~Hz}$, in correspondence to frequencies below $100 \mathrm{~Hz}$, the structural response of the control path is also very low because the excitation is low-pass filtered with a cutoff frequency $F_{c}=480 \mathrm{~Hz}$. These low-pass filters are integrated in order to avoid both control spillover and the step responses of the zero-order hold outputs of the digital-analogue converters. The identified model of the test section of the Dornier 728 has 700 states. It needs to be mentioned that the number of states for the identification of the secondary path model is chosen empirically, focussing on a high correlation between the singular values. Nevertheless, the number of states is far more than needed, so for a more efficient integration, the number of states can be further reduced.

In comparison with the investigations presented by the researchers in [16], the secondary path response in the frequency range of $100 \mathrm{~Hz}$ to $500 \mathrm{~Hz}$ is 


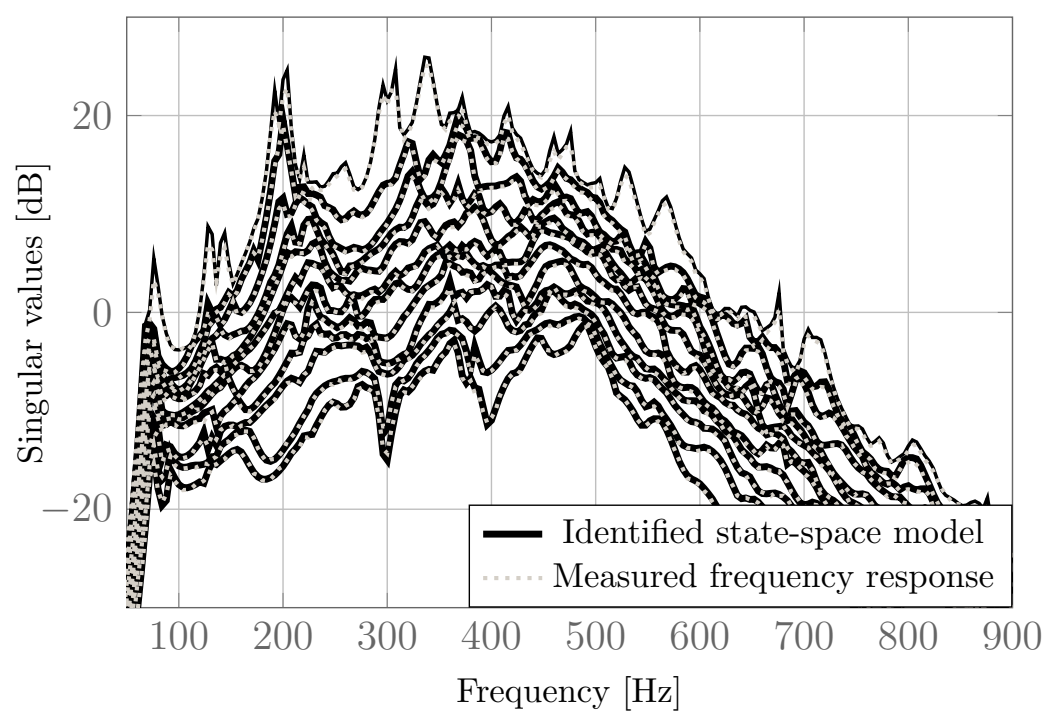

Figure 9: Singular Values of the Dornier 728 model

more flat than the response of the fuselage panel. This is due to the increased damping of a real aircraft fuselage induced by rivets and additional materials. It is important at this point to remember that the Dornier 728 has an aluminium fuselage, which means that the interior noise problems of aircraft with a CFRP fuselage will only increase due to the lower damping of integrally built CFRP and that this further motivates the use of an AVC system.

\subsection{Feedforward control of the test section}

First of all, the vibration patterns of the Dornier 728 with the fully equipped control sections are measured in order to get a reference in the 'Control off' state. Afterwards, two sensor configurations for the active control systems are investigated; a nearly collocated control system $(12 \mathrm{~A} \times 12 \mathrm{~S})$ and a system with a maximal number of sensors $(12 \mathrm{~A} \times 25 \mathrm{~S})$. 'Nearly collocated' means that the sensors are placed geometrically as close as possible to the actuators. A collocated system is not realisable when the sensors are mounted on the same side as the actuators. The nearly collocated system is a quadratic (i.e., equal number of sensors and actuators) system that has a much greater potential to reduce the local error signals than a rectangular system (i.e., more sensors than actuators) [22]. Compared with previous 
measurements presented in [16] the number of sensors and actuators per square meter are reduced in this investigation and therefore, only the first five CROR frequencies are addressed with the control systems.

The local vibration attenuation at the accelerometers of the two control systems is presented in Figure 10a. The collocated control system generates a strong local vibration attenuation of over $30 \mathrm{~dB}$. This is a very impressive local vibration reduction, but it leads to vibration restructuring instead of a reduction, which can be seen when the global vibration reduction and the sound radiation are considered. Therefore, only lesser reductions can be measured, e.g. $5 \mathrm{~dB}$ in global vibration and $1.8 \mathrm{~dB}$ in sound intensity for the first CROR BPF.

The larger spatial observability and therefore a better global performance of the control system with 25 sensors can be seen in the global vibration attenuation, shown in Figure 10b. With only small local vibration reductions the control system with 25 sensors produces a more homogeneous reduction over the whole fuselage surface up to the third CROR frequency at $268.5 \mathrm{~Hz}$.

Above the third CROR BPF, both control systems achieve only local vibration reductions and lead to an amplification in radiated sound intensity, shown in Figure 11. For the collocated control system, this effect is more noticeable and is mainly caused by the pinning effect mentioned in [23], which leads to an efficient restructuring of the vibration patterns in such a way that they radiate sound more efficiently. Furthermore, even the global vibration amplitude is increased due to the pinning effect for the collocated system. In contrast to this, the global vibration is nearly unaffected by the 25 sensor control system whereas the sound intensity, especially for the fifth CROR frequency, is also increased by a vibration restructuring.

At the second CROR BPF, both control systems show an interesting phenomenon. Despite a global vibration reduction, the radiated sound intensity is increased by both control systems. Referring to Figure 13, it can be seen that the vibration pattern is restructured at the window section. In both cases, there is only one residual vibration maximum at the left section that radiates sound more efficiently than the two vibration maxima which vibrate in anti-phase in the uncontrolled case. In contrast to this, the first CROR frequency, shown in Figure 12, is restructured by neither of the control systems and therefore the sound radiation behaviour stays unchanged and the global vibration reduction is correlated to the sound intensity reduction.

An interesting result can also be seen at the third CROR frequency (268.5 $\mathrm{Hz}$ ). Here, a different restructuring occurs (see Figure 14) for the two con- 


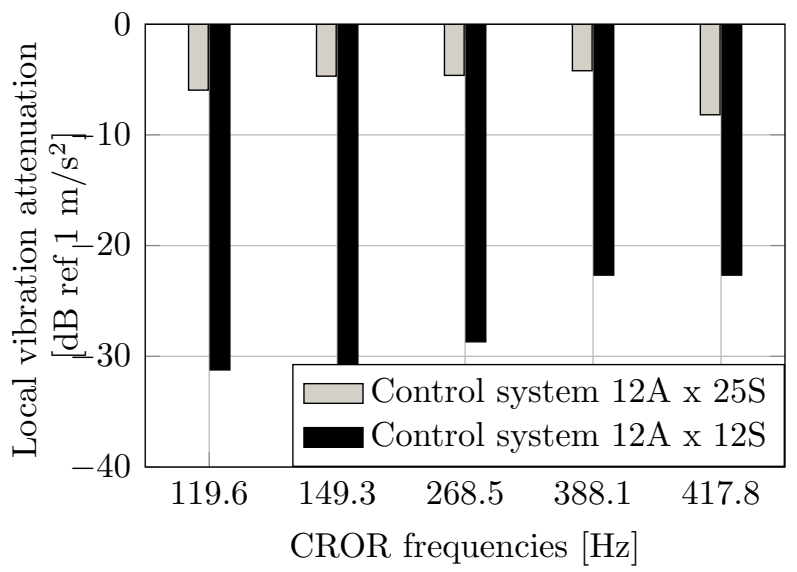

(a) Local vibration

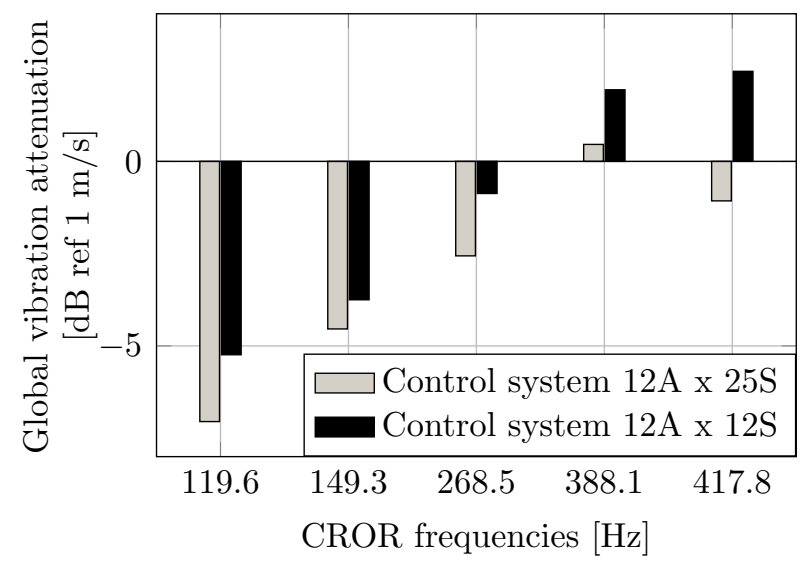

(b) Global vibration

Figure 10: Averaged vibration attenuation at the local error sensors and the LSV sensor grid 


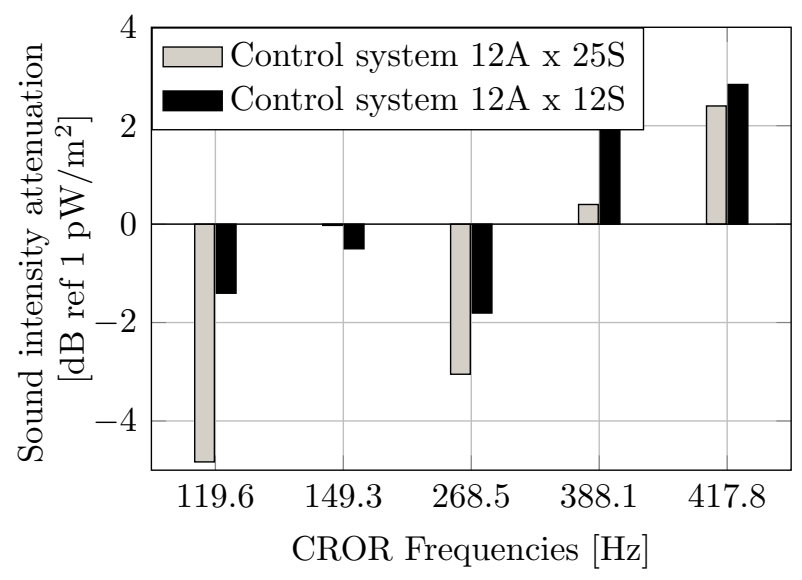

Figure 11: Radiated intensity attenuation of Section 18 and 19

trol systems, but the intensity reduction correlates with the global vibration reduction. The effect of the restructuring and the resulting intensity radiation have to be further investigated because the structure's complexity complicates the understanding of its acoustic short circuit effects.

\subsection{Discussion}

The active feedforward control systems described in this study show a reduction of sound intensity of up to $5 \mathrm{~dB}$ and a global vibration reduction of up to $6 \mathrm{~dB}$ which is significant considering the complexity of the test section. Nevertheless, a lot of further investigations have to be done in order to further improve the performance of the control system.

The acoustic characterisation of the test section has shown that the sound is mainly transmitted towards the lower part of the test section. Considering this aspect and the local impact of the actuators, the actuator placement needs to be improved by a more thorough consideration of the test section's lower part. Furthermore, the number and position of the sensors has a significant influence on the system performance, as becomes apparent considering the restructuring of the vibration patterns and the very strong local vibration reductions. For further investigations, the position of the actuators and the sensors needs to be optimised in such a way that the sound radiation can also be addressed with an active vibration control system.

Even the use of an ASAC system can be considered. But, as has been mentioned in [14], an increase in global vibration, which is typical for an ASAC 

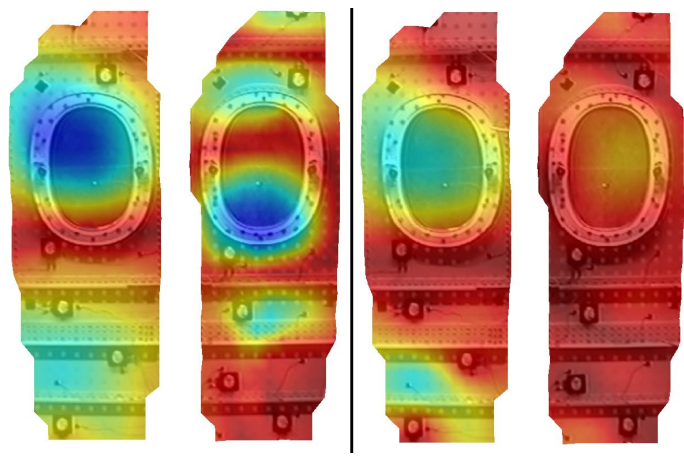

(a) $12 \times 12$ system
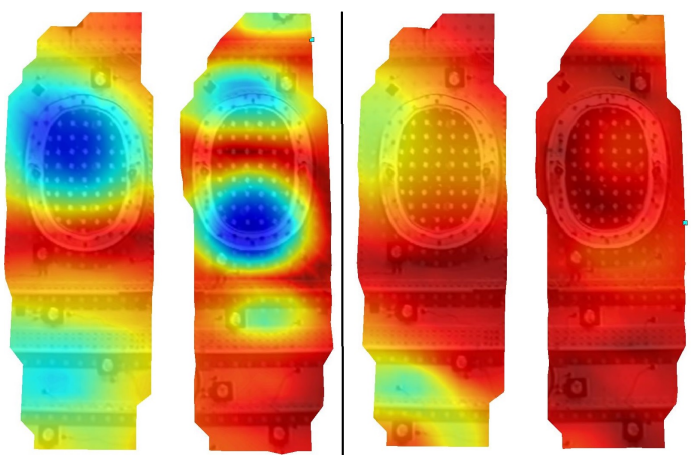

(b) $12 \times 25$ system

Figure 12: Uncontrolled (left) and controlled (right) vibration pattern at frequency $119.6 \mathrm{~Hz}$ for the 12 actuator and 12 sensor system (a) and the 12 actuator and 25 sensor system (b) 

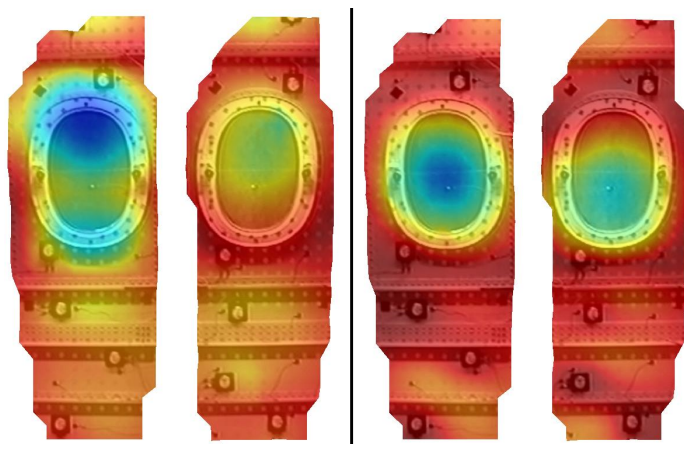

(a) $12 \times 12$ system
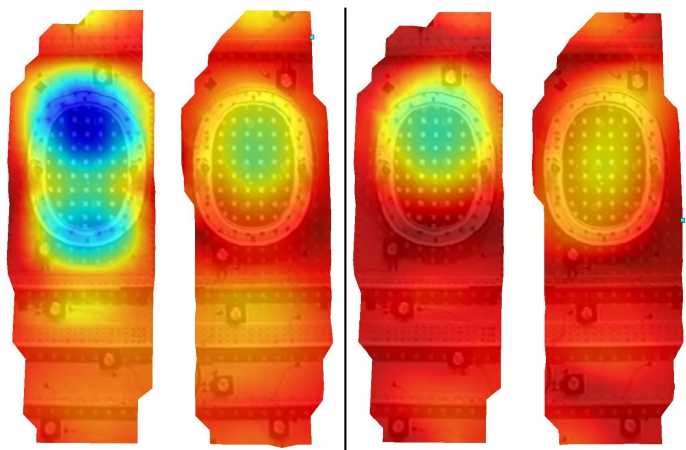

(b) $12 \times 25$ system

Figure 13: Uncontrolled (left) and controlled (right) vibration pattern at frequency $149.3 \mathrm{~Hz}$ for the 12 actuator and 12 sensor system (a) and the 12 actuator and 25 sensor system (b) 

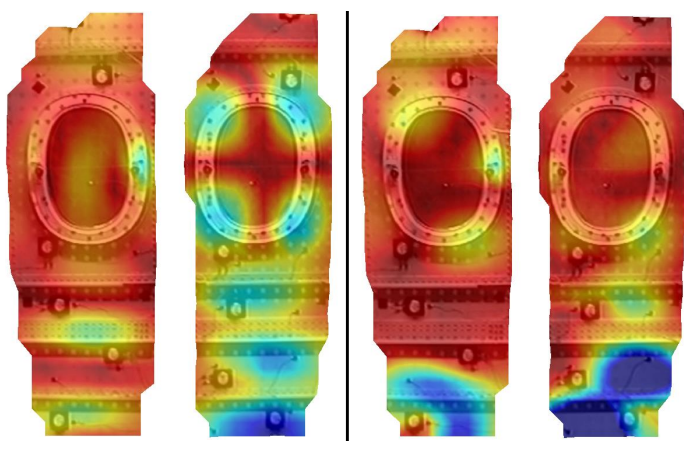

(a) $12 \times 12$ system
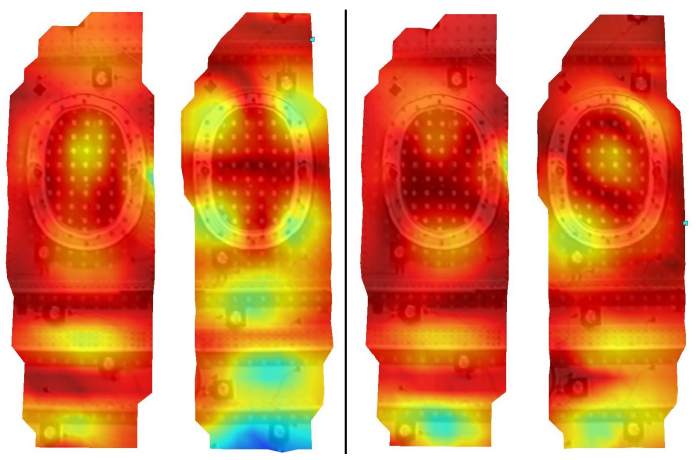

(b) $12 \times 25$ system

Figure 14: Uncontrolled (left) and controlled (right) vibration pattern at frequency $268.5 \mathrm{~Hz}$ for the 12 actuator and 12 sensor system (a) and the 12 actuator and 25 sensor system (b) 
system, should be avoided on a fuselage structure because of fatigue issues. Therefore, an AVC system used for vibration reduction and optimised to influence the sound radiation indirectly appears to be the best choice for a fuselage structure [24].

Apart form these main issues of the study, the actuator coupling into the fuselage structure shows that the actuators induce the vibrations very locally. This could be an advantage for decentralised control systems and should be further investigated if a larger test section is considered.

\section{Conclusions}

This paper presents a preliminary study of the implementation of an active feedforward controller into a typical regional aircraft under a CROR excitation. First of all, typical control actuators for smart structures are tested on the fuselage of the experimental prototype aircraft Dornier 728. With regard to the low frequency noise produced by the CROR engines, the inertial actuators outperform the piezoceramic patch actuators in terms of the magnitude of the frequency response functions, which is why these actuators are considered for the presented study. Yet, the weight penalty of inertial actuators is much higher than that of piezoceramic actuators. For this reason further investigations with multiple piezoceramic actuators should be performed.

The main focus of this paper lays on the experimental test of an active feedforward controller with two different sensor concepts. Firstly, a collocated sensor/actuator (12 sensors and 12 actuators) system is investigated and afterwards compared to a system with 12 actuators and 25 sensors. Similar to the results of the investigations made in [16], the local vibration reductions at the error sensors locations are quite significant for the collocated control system, whereas the overall performance measured in terms of global vibration reduction and sound intensity reduction remains negligible. Compared with the collocated system, the 25-sensor system produces a more homogeneous global vibration reduction, which leads to a sound intensity reduction of up to $5 \mathrm{~dB}$ in the first and $3 \mathrm{~dB}$ in the third CROR frequency. However, there are many ways in which to improve the performance of the feedforward control systems because the restructuring of operational vibration patterns depends on the sensor and actuator placement. By influencing the restructuring the sound radiation can be also significantly increased or decreased. Further investigations need to address the question of how much performance 
can be achieved if the placement of the sensors and actuators is optimised in order to address noise problems with an AVC system. Maybe the critical restructuring of the vibration patterns can be avoided in such a way that a global vibration reduction is directly correlated to a sound intensity reduction.

[1] F. Farassat, S. Padula, M. Dunn, Advanced turboprop noise prediction based on recent theoretical results, Journal of Sound and Vibration 119 (1987) 53-79.

[2] A. Stuermer, J. Yin, Low-speed aerodynamics and aeroacoustics of cror propulsion systems, in: Proc. of 15th AIAA/CEAS Aeronautics Conference.

[3] S. Algermissen, O. Unruh, T. Haase, H. P. Monner, Reduction of rotor noise transmission at the dornier 728 experimental aircraft, in: Proceedings of ICSV.

[4] C. Fuller, C. Rogers, H. Robertshaw, Control of sound radiation with active/adaptive structures, Journal of Sound and Vibration 157 (1992) 19-39.

[5] B. Bingham, M. Atalla, N. Hagood, Comparison of structural-acoustic control designs on an active composite panel, Journal of Sound and Vibration 244 (2001) 761-778.

[6] L. Babu, C. Padmanabhan, Hybrid active and passive noise control of cavities, Acta Acustica united with Acustica 97 (2011) 752-760.

[7] A. Sitel, C. Guigou-Carter, P. Jean, Numerical method for predicting the sound reduction index of a double panel including an active control system and porous material, Acta Acustica united with Acustica 97 (2011) 569-578.

[8] H. Breitbach, D. Sachau, S. Böhme, Acoustic challenges of the a400m for active systems, in: Smart Structures and Materials, International Society for Optics and Photonics, pp. 617104-617104.

[9] R. Cabell, D. Palumbo, J. Vipperman, A principal component feedforward algorithm for active noise control: Flight test results, Control Systems Technology, IEEE Transactions on 9 (2001) 76-83. 
[10] B. Petitjean, I. Legrain, F. Simon, S. Pauzin, Active control experiments for acoustic radiation reduction of a sandwich panel: Feedback and feedforward investigations, Journal of Sound and Vibration 252 (2002) 19-36.

[11] E. Bianchi, P. Gardonio, S. Elliott, Smart panel with multiple decentralized units for the control of sound transmission. part iii: control system implementation, Journal of sound and vibration 274 (2004) 215-232.

[12] M. Misol, S. Algermissen, H. Monner, Experimental investigation of different active noise control concepts applied to a passenger car equipped with an active windshield, Journal of Sound and Vibration (2012).

[13] O. Heintze, M.Misol, S. Algermissen, C. Hartung, Active structural acoustic control for a serial production truck oil pan: Experimental realization, in: Proceedings of Adaptronic Congress.

[14] P. Gardonio, Review of active techniques for aerospace vibro-acoustic control, Journal of aircraft 39 (2002) 206-214.

[15] A. Grewal, D. Zimcik, L. Hurtubise, B. Leigh, Active cabin noise and vibration control for turboprop aircraft using multiple piezoelectric actuators, Journal of intelligent material systems and structures 11 (2000) 438-447.

[16] T. Haase, S. Algermissen, O. Unruh, M. Misol, Experiments on active control of counter-rotating open rotor interior noise, Acta Acustica united with Acustica 100 (2014) 448-457.

[17] S. Algermissen, S. Meyer, C. Appel, H. Monner, Experimental synthesis of sound pressure fields for active structural acoustic control testing, in: Proc. of 23rd International Conference on Adaptive Structures and Technologies (ICAST), Nanjing (China).

[18] S. M. Kuo, D. R. Morgan, Active noise control system: algorithms and DSP implementations, John Wiley \& Sons, INC., 1.aufl. edition, 1996.

[19] S. Elliott, Signal processing for active control, Academic Press, 1.aufl. edition, 2001. 
[20] A. Jessop, K. Li, J. Bolton, Reduction of low frequency noise transmitted through a single-pane window, Acta Acustica united with Acustica 97 (2011) 382-390.

[21] T. Katayama, Subspace methods for system identification, Springer, 2005.

[22] J. Minkoff, The operation of multichannel feedforward adaptive systems, Signal Processing, IEEE Transactions on 45 (1997) 2993-3005.

[23] P. Gardonio, E. Bianchi, S. Elliott, Smart panel with multiple decentralized units for the control of sound transmission. part i: theoretical predictions, Journal of sound and vibration 274 (2004) 163-192.

[24] T. Haase, C. Schubert, H. P. Monner, Active vibration control systems with improved sound radiation properties, in: Proceedings of ICAST. 